\title{
Frequency-Offset Estimation for HIPERLAN
}

\author{
C. Tellambura, Member, IEEE, I. R. Johnson, Y. J. Guo, and S. K. Barton, Senior Member, IEEE
}

\begin{abstract}
Frequency-offset correction is considered for a HIPERLAN (HIgh-PErformance Radio LAN) system over the indoor radio channel. Since the multipath channel response is not known a priori, a viable frequency-offset estimator should not depend on such knowledge. Such an estimator, using a single sample per symbol, is derived for HIPERLAN. The estimator is shown to approach the Cramer-Rao bound for frequency-offset estimation over a multipath channel. A HIPERLAN system simulation example shows that the performance with an offset of $150 \mathrm{kHz}$ is within $0.5 \mathrm{~dB}$ of that of a system with zero frequency offset.
\end{abstract}

Index Terms-Frequency-offset estimation, indoor radio communication, maximum-likelihood estimation, multipath channels.

\section{INTRODUCTION}

$\mathbf{T}$ HE HIPERLAN (HIgh PErformance Radio LAN) standard [1] specifies a wireless LAN system designed to support user data rates up to $23.5 \mathrm{Mbit} / \mathrm{s}$ in indoor channels. The severe intersymbol interference (ISI) affecting transmission at this data rate and the frequency offset (FO) of local oscillators at $5 \mathrm{GHz}$ hamper the performance. An adaptive equalizer can be used to mitigate the ISI, but it cannot follow the channel variations caused by the FO, which can be up to $200 \mathrm{kHz}$ [2]. To compensate for the FO, a data-directed phase-locked loop can be employed [2], which is sensitive to the multipath delay spread.

The HIPERLAN standard specifies a 450-bit-long periodic training sequence (TS)

$$
m_{1} m_{1} m_{1} m_{2} m_{2} m_{2} m_{3} \cdots m_{5} m_{5} m_{5}^{\prime}
$$

where each $m_{k}$ is a 31-bit $m$-sequence and $m_{\tilde{5}}^{\prime}$ is a 16-bit segment of $m_{5}$ [1]. Exploiting this cyclic structure, this paper derives a feedforward FO estimator which does not require knowledge of the transmission channel. It is a maximumlikelihood estimator (MLE) and is conditionally unbiased for a high signal-to-noise ratio (SNR).

The basic idea is as follows. Since the contribution to the signal phase of the symbol within a period is $-\pi / 2$, comparing

Paper approved by E. Panayirci, the Editor for Synchronization and Equalization of the IEEE Communications Society. Manuscript received March 15, 1997; revised June 30, 1998 and November 15, 1998. This work was carried out at the University of Bradford and supported by the UK DTI/EPSRC LINK Project PC2011 "High Throughput Radio Modem" under EPSRC Grant GR/K 00318 in collaboration with Symbionics Networks Ltd.

C. Tellambura is with the Communications and DSP Research Group, School of Computer Science and Software Engineering, Monash University, Clayton, Vic. 3168, Australia (e-mail: chintha@dgs.monash.edu.au).

I. R. Johnson is with Hewlett-Packard Laboratories, Stoke Gifford, Bristol BS12 6QZ U.K.

Y. J. Guo is with Fujitsu Europe Telecom R\&D Center Ltd. (FTRC), Stockley Park, Uxbridge UB11 1AB U.K.

S. K. Barton is with Institute of Integrated Information Systems, School of Electronic and Electrical Engineering, University of Leeds, Leeds LS2 9JT U.K

Publisher Item Identifier S 0090-6778(99)06292-3. the phases of two samples yields the phase contribution due to FO in the same period. This requires that the radio channel be constant for the packet duration and the length of the channel impulse response be less than 31 samples. These are reasonable assumptions. Further, clock recovery issues are not considered in the paper. The algorithm uses samples that are taken at the symbol rate.

\section{Frequency OfFSET Estimation}

\section{A. System Model}

The channel is modeled as a linear filter with $\underline{h}=$ $\left(h_{0}, h_{1}, \cdots, h_{M-1}\right)$, where $M$ is the number of multipath components, $h_{k}$ is a zero-mean independent complex Gaussian random variable. In indoor channels, the Doppler spread is very small, so the channel taps $\left\{h_{k}\right\}$ are assumed constant during a frame which can last about $1 \mathrm{~ms}$ at most (i.e., 25000 bits). Further, $M<31$ is assumed. Since $T=42.5$ ns and measurements show that some indoor channels have rms delay spreads of $50 \mathrm{~ns}$ or less, this assumption is reasonable. Even for a worst case rms delay spread of $150 \mathrm{~ns}$, the worst case delay window may be about 450 ns (i.e., $M<10$ ).

A HIPERLAN-compliant modem uses Gaussian minimum shift keying (GMSK) modulation with $B T$ product of 0.3 [1]. GMSK is obtained by applying filtered data to a frequency modulator. The complex output of the GMSK modulator $s(t, \underline{x})$ depends on the total phase $\phi(t, \underline{x})$ where $\underline{x}=$ $\left\{x_{0}, x_{1}, \cdots\right\}$ is the input data sequence. If $s_{n} \triangleq s(n T, \underline{x})$, one has

$$
s_{n+31}=-j s_{n}
$$

when $\underline{x}$ is the TS. Let $\theta_{n}$ be the phase state, the accumulated phase of all the previous symbols that have passed through the filter. Thus, $\theta_{n+31}-\theta_{n}=-\pi / 2$ since the sum of $\left\{x_{i}\right\}$ over a 31-bit interval is -1 . Hence, (1) follows.

For the noiseless and zero FO case, the channel output is the linear convolution between $\underline{h}$ and $\left\{s_{n}\right\}$. Given (1), it follows that $y_{n+31}=-j y_{n}$ for $n>n_{0}$ where $n_{0}$ is selected to avoid the transient period of the output. The received samples can be expressed as

$$
r_{n}=y_{n} e^{j\left(n \delta+\phi_{0}\right)}+v_{n}
$$

where $v_{n}$ is a complex Gaussian noise sample (zero mean and variance $\left.\sigma^{2}\right), \delta=2 \pi \Delta F T$ is the $\mathrm{FO}$ in radian/bit, $\Delta F$ is the FO in hertz, and $\phi_{0}$ is a random initial phase. 


\section{B. Extraction of Frequency-Offset Estimate}

Let

$$
\begin{aligned}
\underline{y} & \left.=\left[y_{n_{0}} e^{j\left(n_{0} \delta+\phi_{0}\right)}, \cdots, y_{n_{0}+30} e^{j\left[\left(n_{0}+30\right) \delta+\phi_{0}\right.}\right]\right]^{\prime} \\
\underline{r}_{0} & =\left[r_{n_{0}}, \cdots, r_{n_{0}+30}\right]^{\prime}, \quad \underline{r}_{1}=\left[r_{n_{0}+31}, \cdots, r_{n_{0}+61}\right]^{\prime} \\
\underline{v}_{0} & =\left[v_{n_{0}}, \cdots, v_{n_{0}+30}\right]^{\prime} \\
\underline{v}_{1} & =\left[v_{n_{0}+31}, \cdots, v_{n_{0}+61}\right]^{\prime}
\end{aligned}
$$

where ' denotes transpose of a row vector. Thus, a set of 62 received samples can be expressed as $\underline{r}_{0}=y+\underline{v}_{0}$ and $\underline{r}_{1}=-j \underline{y} e^{j 31 \delta}+\underline{v}_{1}$.

Let $z \triangleq j \underline{r}_{0}^{*} \underline{r}_{1}$ where the asterisk denotes conjugate transpose. The sum is taken over 31 samples of $r_{n}^{*} r_{n+31}$. This summing increases the SNR of the estimate. Following a similar case [3], it can be shown that the MLE is

$$
\hat{\delta}=[\arg z] / 31 \text {. }
$$

$\arg z$ is a multivalued function unless $-\pi<\arg z \leq \pi$. Thus, $\delta$ is unambiguously estimated if $|\delta|<\pi / 31$ with a corresponding maximum FO $\Delta F$ given by $2 \pi|\Delta F| T<\pi / 31$. In HIPERLAN, this corresponds to about $379 \mathrm{kHz}$.

\section{Statistical Properties of the MLE for Weak Noise}

If the noise terms are small, then

$$
z \approx\|\underline{y}\|^{2} e^{j 31 \delta}+\underline{y}^{j 31 \delta} \underline{v}_{0}^{*}+j \underline{v}_{1} \underline{y}^{*}
$$

where the vector norm $\|\underline{y}\|=\sqrt{\underline{y y^{*}}}$. The noise term $\underline{v}_{1} \underline{v}_{0}^{*}$ has been neglected. Hence, $z$ is complex Gaussian with mean and variance: $m_{z}=\|y\|^{2} e^{j 31 \delta}$ and $\sigma_{z}^{2}=2 \sigma^{2}\|y\|^{2}$. Thus, the estimator (3) is conditionally unbiased under weak-noise conditions. Further, if $z=|z| e^{j \hat{\theta}}$ and $\epsilon=\hat{\theta}-31 \delta$, then the probability density function (pdf) of the error $\epsilon$ can be easily derived using the well-known pdf of the phase of a noisy sinusoid [4, p. 270]

$$
f(\epsilon)=\frac{e^{-\gamma / 2}}{2 \pi}+\sqrt{\frac{\gamma}{8 \pi}} e^{-\gamma \sin ^{2} \epsilon / 2} \operatorname{erfc}\left\{-\sqrt{\frac{\gamma}{2}} \cos \epsilon\right\} \cos \epsilon
$$

where the $\operatorname{SNR} \gamma \triangleq\|y\|^{2} / \sigma^{2}$ and $\operatorname{erfc}(x)$ is the complementary error function. This pdf can be used to compute the mean square value

$$
\overline{\epsilon^{2}}=\int_{-\pi}^{\pi} \epsilon^{2} f(\epsilon) d \epsilon
$$

which yields the variance of $\hat{\delta}, \operatorname{var}(\hat{\delta})$.

The Cramer-Rao bound (CRB) is a lower bound on the mean-square error of any unbiased estimate [5]. To derive the CRB, (2) is rewritten as $r_{n}=a_{n} e^{j\left(\varphi_{n}+n \delta\right)}+v_{n}$ where $a_{n}=\left|y_{n}\right|$ and $\varphi_{n}=\arg y_{n}+\phi_{0}$. It is required to estimate $\delta$ using 62 samples of $r_{n}$, but $\left\{a_{n}\right\}$ and $\left\{\varphi_{n}\right\}$ are unknown and unwanted. Called nuisance parameters [5], their presence reduces information on $\delta$ and increases the variance bound. In the derivation of $\mathrm{CRB},\left\{a_{n}\right\}$ and $\left\{\varphi_{n}\right\}$ are regarded as unknown deterministic parameters.

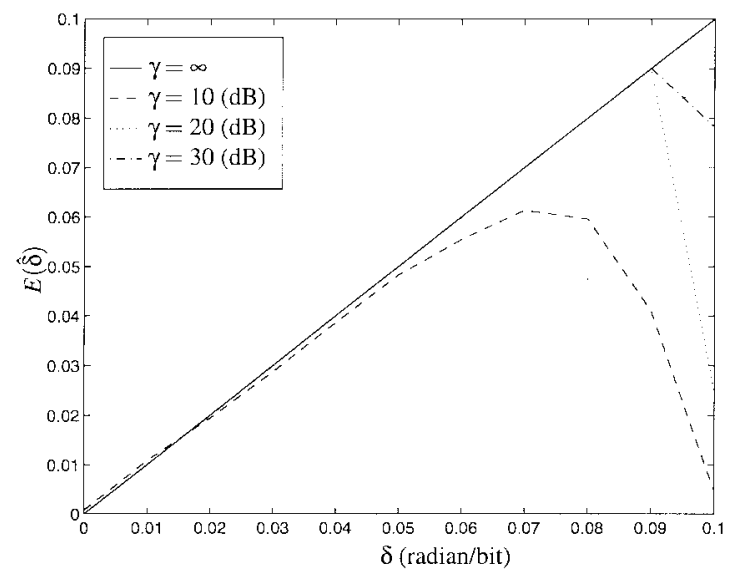

Fig. 1. Expected value of the frequency-offset estimate $\hat{\delta}$ versus the actual FO $\delta(E(\hat{\delta})$ by simulation).

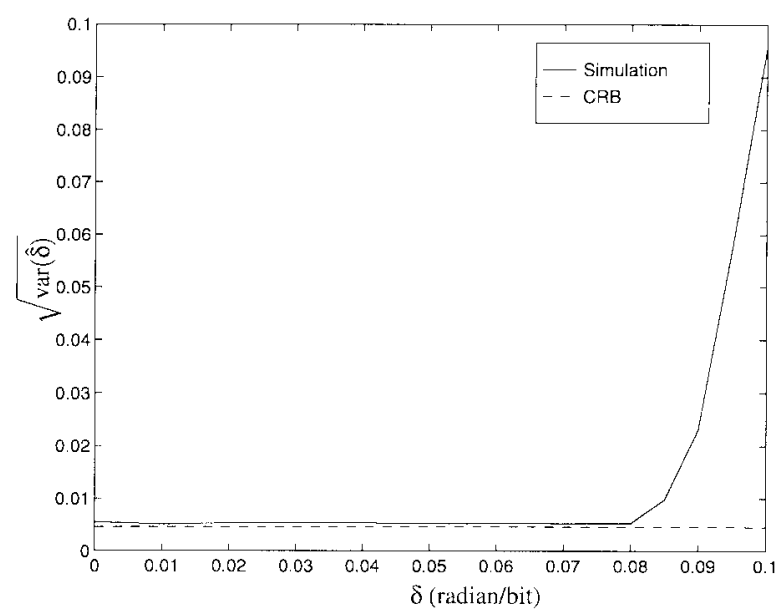

Fig. 2. SD of the frequency-offset estimate $\hat{\delta}$ versus the actual FO $\delta$. SNR is given by $\gamma=17 \mathrm{~dB}$.

Since 31 distinct pairs of $\left(a_{n}, \varphi_{n}\right)$ exist, let $\underline{\theta}=$ $\left\{a_{n_{0}}, \cdots, a_{n_{0}+30}, \varphi_{n_{0}}, \cdots, \varphi_{n_{0}+30}, \delta\right\}$. Thus, the Fischer information matrix $J(\underline{\theta})$ is a $63 \times 63$ matrix. The $\log$ likelihood function is

$$
\begin{aligned}
\log f_{\underline{\theta}}(y)=-\frac{1}{\sigma^{2}} & \sum_{n=n_{0}}^{n_{0}+61}\left|r_{n}\right|^{2}+a_{n}^{2} \\
& -r_{n} a_{n} e^{-j\left(\varphi_{n}+n \delta\right)}-r_{n}^{*} a_{n} e^{j\left(\varphi_{n}+n \delta\right)} .
\end{aligned}
$$

Entries in $J(\underline{\theta})$ can now be computed using the appropriate partial derivatives of (5). Thus, the $\mathrm{CRB}$ for $\delta$ is [63rd diagonal element of the inverse of $J(\underline{\theta})]$

$$
\operatorname{var}(\hat{\delta}) \geq\left(J^{-1}(\underline{\theta})\right)_{63,63} .
$$

\section{RESUlTS AND CONCLUSIONS}

For Figs. $1-4, M=8$ and the channel tap weights are randomly generated with $E\left|h_{k}\right|^{2}=e^{-\alpha k}$ (see Section II-A). Sixty-two samples are used for estimations. The quality of the estimate depends primarily on the energy content $\|y\|^{2}$. In Fig. 4 , the SNR $\bar{E}_{b} / N_{0}$ is defined as $E\left(\|y\|^{2}\right) / 2 \sigma^{2}$ where $2 \sigma^{2}$ is the variance of complex additive white Gaussian noise. 


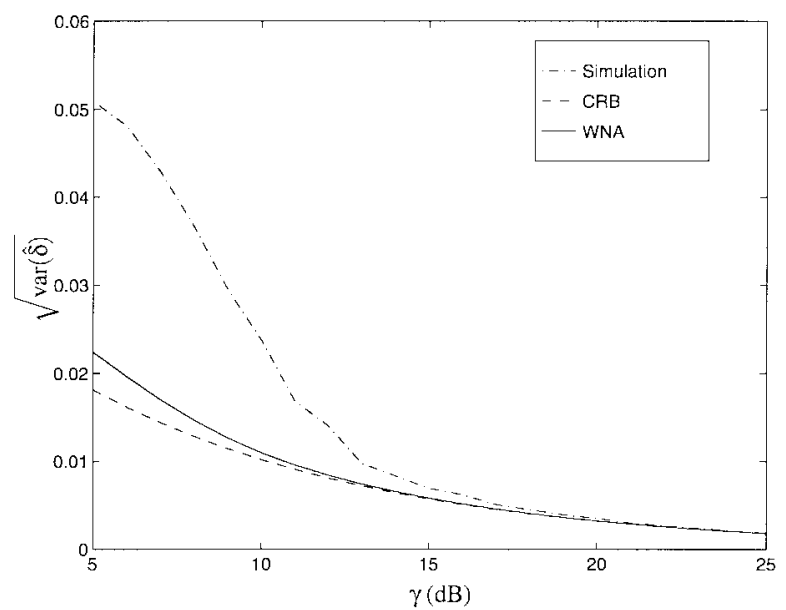

Fig. 3. SD of the frequency-offset estimate $\hat{\delta}$ versus SNR. For simulation results, $\delta=0.05$. Weak noise analysis is based on (5) and (6).

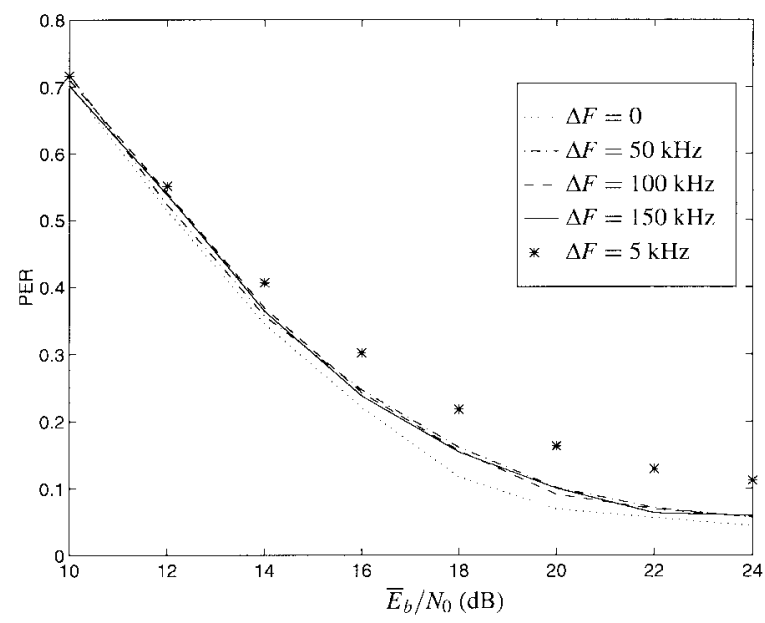

Fig. 4. PER for the equalized system for several values of FO. For $\Delta F=0$ and $5 \mathrm{kHz}$, frequency-offset correction is not employed. Normalized delay spread $\sigma_{n}=0.5$.

Fig. 1 shows the expected value of the frequency estimate versus the FO. Performance for $\gamma \geq 20 \mathrm{~dB}$ is identical to that for $\gamma=\infty$, except for $\delta>0.09$ when frequency aliasing occurs due to the noise (however small). At $\gamma=10 \mathrm{~dB}$, frequency aliasing begins at $\delta=0.05$. This figure is almost half of the theoretical maximum $\pi / 31$. Fig. 2 shows the standard deviation (SD) of the estimate and the CRB. For $\delta<0.08$, the estimator performs quite close to the CRB. Fig. 3 shows the SD versus the SNR $\gamma$. For $\gamma \geq 20 \mathrm{~dB}$, the performance of the estimator reaches the CRB.

The simulated system comprises GMSK $(\mathrm{BT}=0.3)$ with precoding and $(31,26) \mathrm{BCH}$ code (single-error correcting) with block interleaving. The channel is stationary over a packet of 10370 bits. Average system performance is obtained over 1000 randomly generated channels. A decision-feedback equalizer with 11 forward taps and 7 feedback taps is used for reducing ISI, and its tap weights are continuously updated throughout the entire packet (step size 0.01). The samples fed to the equalizer are $r_{n} e^{-j n \hat{\delta}} n=1,2, \cdots$ where $\hat{\delta}$ is the estimated FO using the initial 450 samples corresponding to the training sequence. Thus, the actual FO seen by the equalizer is $\delta-\hat{\delta}$.

Fig. 4 shows several packet error rate (PER) (i.e., the fraction of packets in error after decoding) curves for the system described above. For an FO of $150 \mathrm{kHz}$, the use of FO correction suffers negligible performance degradation relative to the zero-offset case. However, it is not true without FO correction (i.e., $r_{n}$ is fed to the equalizer, not $r_{n} e^{-j n \hat{\delta}}$ ). For instance, at $\bar{E}_{b} / N_{0}=17 \mathrm{~dB}$ and $\Delta F=5 \mathrm{kHz}$, the degradation is about $3 \mathrm{~dB}$. Thus, if uncorrected, even small amounts of FO (several kilohertz) will substantially degrade the system performance. This is due to the tap rotation property of an equalizer [6]; for a FO $\epsilon$, the optimum tap coefficient vector is given by

$$
C_{n \text { opt }}=C_{\mathrm{opt}} \exp [-j 2 \pi n \epsilon]
$$

That is, $C_{n \text { opt }}$ "rotates" with frequency $\epsilon$. Since the step size $\mu$ must be small to keep the steady-state mean-square error small, it is not possible simultaneously to equalize the channel and to rotate the tap coefficients by $2 \pi \epsilon$ radians per symbol interval even for small FO's. With the use of forward frequency-offset correction, the equalizer only has to deal with the residual offset. Fig. 4 shows almost identical performance curves for $\Delta F=50,100$ and $150 \mathrm{kHz}$. This is due to the residual FO being roughly independent of the FO (Fig. 2).

In conclusion, frequency-offset estimation for HIPERLAN has been considered. The effect of FO has been successfully mitigated using a feedforward estimator, which exploits the cyclic structure of the training sequence. This allows errorfree reception of over $90 \%$ of packets over dispersive $(0.5 \leq$ $\left.\sigma_{n} \leq 1.0\right)$ and slowly time-varying channels even for large amounts of FO (e.g., $150 \mathrm{kHz}$ ). Note that the $10 \%$ packet error rate is mainly caused by the frequency selective fading in the channel, not by the FO.

\section{REFERENCES}

[1] ETSprETS 300-652, "Radio Equipment and Systems (RES): High Performance Radio Local Area Network (HIPERLAN) Functional specification," Apr. 1996.

[2] J. Tellado, E. Kayata, and J. M. Cioffi, "Adaptive DFE for GMSK in indoor channels," IEEE J. Select. Areas Commun., vol. 14, pp. 492-501, Apr. 1996.

[3] P. H. Moose, "A technique for orthogonal frequency division multiplexing frequency offset correction," IEEE Trans. Commun., vol. 42, pp. 2908-2914, Oct. 1994.

[4] J. G. Proakis, Digital Communications, 3rd ed. New York: McGraw Hill, 1995.

[5] L. L. Scharf, Statistical Signal Processing. Reading, MA: AddisonWesley, 1991.

[6] R. D. Gitlin, E. Y. Ho, and J. E. Mazo, "Passband equalization of differentially phase-modulated data signals," Bell Syst. Tech. J., vol. 52, pp. 219-238, Feb. 1972. 